\title{
COMPARATIVO DA CINÉTICA DE DECOMPOSIÇÃO TÉRMICA DA PALHA DE CANA-DE-AÇÚCAR EM ATMOSFERA OXIDANTE E INERTE
}

\author{
L. A. HIGUCHI, Y. J. RUEDA-ORDÓÑEZ e K. TANNOUS \\ Universidade Estadual de Campinas, Faculdade de Engenharia Química \\ E-mail para contato: katia@feq.unicamp.br
}

\begin{abstract}
RESUMO - Este trabalho teve como objetivo estudar a cinética de decomposição térmica da palha de cana-de-açúcar através da técnica de termogravimetria. As condições aplicadas foram: partículas de $510 \mu \mathrm{m}$, atmosfera de ar sintético e taxa de aquecimento de $10{ }^{\circ} \mathrm{C} / \mathrm{min}$. A análise cinética foi realizada aplicando o modelo de reações paralelas e independentes considerando três principais componentes da biomassa (celulose, hemicelulose e lignina). Seis reações foram assumidas, sendo três relacionadas à devolatilização de cada componente, e outras três à combustão do carvão remanescente. Os resultados obtidos a partir da modelagem ajustaram de maneira bastante satisfatória aos dados experimentais $\left(\mathrm{R}^{2}=0,9848\right)$. Os parâmetros cinéticos obtidos em atmosfera oxidante foram comparados com dados de literatura em atmosfera inerte, sendo maiores devido ao aumento da temperatura causado pelas reações de oxidação dos voláteis liberados.
\end{abstract}

\section{INTRODUÇÃO}

No Brasil, o aproveitamento energético da biomassa é através do processo de combustão, embora outros processos como pirólise e gaseificação estejam em fase piloto, visando uma futura implementação industrial. A combustão de biomassa é realizada para geração de vapor para uso em turbinas e consequente geração de energia elétrica. No entanto, é ainda um processo ineficiente no qual o conteúdo energético da biomassa não é aproveitado totalmente (Rueda-Ordóñez e Tannous, 2014).

Por conta disso, a pirólise tem sido amplamente estudada, sendo este processo a decomposição térmica da biomassa em ausência de oxigênio, gerando produtos na forma de gases, líquidos e sólidos (Basu, 2010). Apesar de haver diversos estudos sobre a pirólise em atmosfera inerte $\left(\mathrm{N}_{2}, \mathrm{He}, \mathrm{Ar}\right)$, em aplicações práticas, a termodecomposição geralmente ocorre sob a presença de oxigênio (Senneca et al., 2004; Amutio et al., 2012). Neste caso a decomposição dos materiais lignocelulósicos torna-se mais complexa, aumentando a degradação a baixas temperaturas e promovendo o aparecimento de reações entre voláteis e o oxigênio, além de ocorrer posteriormente a combustão do carvão formado nas etapas iniciais (Bilbao et al., 1997).

A cinética da decomposição térmica de biomassa é comumente analisada por termogravimetria e, segundo Órfão et al. (1999), o modelo cinético que melhor representa este processo é o das reações paralelas e independentes. No caso da decomposição térmica em 
atmosfera oxidante este modelo assume que cada componente da biomassa (hemicelulose, celulose e lignina) reage de maneira independente numa faixa de temperatura, e que o somatório dessas reações reproduz a reação da biomassa. Segundo Anca Couce et al. (2012), a hemicelulose libera os voláteis na faixa de temperatura entre $200{ }^{\circ} \mathrm{C}$ e $300{ }^{\circ} \mathrm{C}$, a celulose entre $250{ }^{\circ} \mathrm{C}$ e $350{ }^{\circ} \mathrm{C}$, e a lignina entre $200{ }^{\circ} \mathrm{C}$ e $500^{\circ} \mathrm{C}$, e o resíduo carbonoso se oxida entre $400{ }^{\circ} \mathrm{C}$ e $600{ }^{\circ} \mathrm{C}$.

Com o intuito de obter uma descrição detalhada da reação de decomposição térmica da palha de cana-de-açúcar, foi realizado um estudo comparativo da cinética deste processo em atmosfera oxidante e inerte aplicando o método das reações paralelas e independentes.

\section{MATERIAIS E MÉTODOS}

Neste trabalho foi utilizada a palha de cana-de-açúcar (Saccharum officinarum L.), moída em moinho de martelo (Tigre S.A., CV2, Brasil) e separada por peneiramento (peneiras Produtest) para obtenção do diâmetro médio de partícula (diâmetro de Sauter) de $510,31 \mu \mathrm{m}$. A umidade inicial da biomassa foi de $7 \%$ determinada através da norma ASTM E871-82.

\subsection{Análise Termogravimétrica}

Para a obtenção das curvas de termogravimetria (TG) e de sua derivada (DTG), utilizou-se um analisador termogravimétrico (Shimadzu, Modelo TGA-50, Japão), capaz de avaliar perdas de massa na ordem de $0,001 \mathrm{mg}$. Para tal foi utilizado um porta-amostra de alumina $(\mathrm{V}=8 \mu \mathrm{L})$ e $3,022 \mathrm{mg}$ de biomassa. As análises foram realizadas em atmosfera de ar sintético (White Martins, $X\left(\mathrm{O}_{2}\right)=20,00 \pm 0,05 \%$ e $X\left(\mathrm{~N}_{2}\right)=80,00 \pm 0,05 \%$ ) à vazão de $50 \mathrm{~mL} / \mathrm{min}$ e faixa de temperatura do meio de $26,8{ }^{\circ} \mathrm{C}$ até $900^{\circ} \mathrm{C}$. A temperatura foi controlada à taxa de $10{ }^{\circ} \mathrm{C} /$ min registrando medidas de massa a cada $5 \mathrm{~s}$.

Os dados experimentais em atmosfera inerte (nitrogênio) (White Martins, $X\left(\mathrm{~N}_{2}\right)=$ 99,996\%, grau 4.6 FID), foram obtidos por Rueda-Ordóñez e Tannous (2014) trabalhados nas mesmas condições acima citadas. Os resultados foram usados como análise comparativa da decomposição da biomassa.

\subsection{Metodologia da Modelagem Cinética}

Inicialmente, os dados experimentais foram transformados em massa normalizada $(W)$ conforme a Equação 1, em que $m_{t}$ representa a massa em uma determinada temperatura e $m_{i}$ representa a massa inicial. Utilizando a Equação 2, calculou-se a derivada da massa normalizada (DTG normalizado), representada por $d W / d t$.

$$
\begin{aligned}
& W=m_{t} / m_{i} \\
& (d W / d t)=d m_{t} / d t\left(1 / m_{i}\right)
\end{aligned}
$$

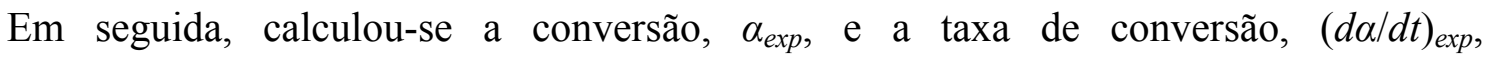
experimentais por meio da Equação 3 e da Equação 4, respectivamente. Nessas equações, $m_{f}$ representa a massa de biomassa presente ao final do experimento. 


$$
\begin{aligned}
& \alpha_{\text {exp }}=\left(m_{i}-m_{t}\right) /\left(m_{i}-m_{f}\right) \\
& (d \alpha / d t)_{\exp }=\left(-d m_{t} / d t\right)\left[1 /\left(m_{i}-m_{f}\right)\right]
\end{aligned}
$$

Pela Equação 5, obtêm-se as taxas de conversão $(d \alpha / d t)$ modeladas para cada componente, seguindo a lei de Arrhenius. Nesta equação $A$ representa o fator pré-exponencial, $E$ a energia de ativação, $n$ a ordem de reação, $R$ a constante universal dos gases, $T$ a temperatura absoluta e $\alpha_{t e o}$, a conversão teórica. Através do método de Runge-Kutta de quarta ordem, calculou-se a conversão teórica, $\alpha_{t e o}$,

$$
(d \alpha / d t)_{l}=A\left(1-\alpha_{t e o}\right)^{n} \exp [-E /(R T)]
$$

A Equação 6 corresponde ao método das reações paralelas e independentes, a qual se aplica às taxas de conversão individuais (Equação 5) e as frações de cada componente (hemicelulose, celulose, lignina). Estas frações estão representadas por $F$ e o subscrito $i$, esta relacionado a cada reação assumida. Neste estudo foram assumidas seis reações, sendo três relacionadas à devolatilização de cada componente, e outras três à combustão do carvão remanescente. Para obtenção dos parâmetros cinéticos $(E$ e $A)$ foi aplicado o método dos mínimos quadrados, onde as taxas de conversão experimental e teórica foram comparadas.

$$
(d \alpha / d t)_{\text {teórico }}=\sum_{i}^{6} F_{i}(d \alpha / d t)_{i}=
$$

Para avaliar a qualidade do ajuste dos dados das taxas de conversão teórica com a experimental, calculou-se o coeficiente de determinação não linear, $R^{2}$, sendo aceitos valores superiores a 0,9800. O software MS Office Excel 2007 (12.0.6683.5002) foi utilizado para realizar a análise dos dados experimentais e aplicação do método cinético das reações paralelas e independentes.

\section{RESULTADOS E DISCUSSÃO}

\subsection{Análise da Decomposição Térmica}

Na Figura 1 estão apresentadas a decomposição térmica da biomassa na forma de massa normalizada e sua derivada em função da temperatura. Na curva de massa normalizada referente à atmosfera oxidante, é possível observar que, inicialmente, há uma perda de massa na região de 25 a $100{ }^{\circ} \mathrm{C}$ associada à perda de umidade pela biomassa, cerca de $8 \%$ da massa inicial. A hemicelulose, por ser mais instável, é decomposta a temperaturas mais baixas $\left(150^{\circ} \mathrm{C}-350^{\circ} \mathrm{C}\right)$, representada pelo "ombro" na curva de DTG, e a celulose entre 275 e $350{ }^{\circ} \mathrm{C}$ representada pelo pico maior. A maior perda de massa entre estas faixas de temperatura (250$350{ }^{\circ} \mathrm{C}$ ), com $66 \%$ da massa inicial e representada pelo maior pico da curva de DTG normalizada $\left(\mathrm{T} \sim 338{ }^{\circ} \mathrm{C}\right)$. Essa grande perda de massa é devida à liberação de materiais voláteis provenientes da decomposição térmica da hemicelulose e da celulose. A lignina, por apresentar maior estabilidade devido a seu conteúdo aromático, decompõe-se lentamente em uma larga faixa de temperatura entre 250 e $500{ }^{\circ} \mathrm{C}$.

Também na Figura 1, compararam-se os resultados obtidos em atmosfera oxidante com atmosfera inerte (Rueda-Ordóñez e Tannous, 2014). Observa-se o efeito da presença de 


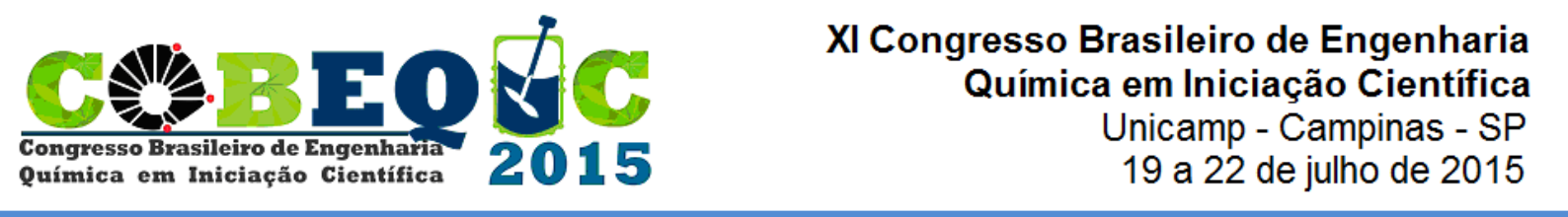

oxigênio na ocorrência do pico de maior taxa de perda de massa na temperatura de $340^{\circ} \mathrm{C}, 50$ ${ }^{\circ} \mathrm{C}$ mais baixa do que o pico em atmosfera inerte $\left(390^{\circ} \mathrm{C}\right)$, e com amplitude 1,8 vezes maior. Este efeito é devido às interações entre os reagentes e os produtos volatilizados, as quais produzem reações de oxidação incrementando a velocidade da reação, e diminuindo a temperatura de reação, o qual não acontece com atmosfera inerte. Na temperatura de $435{ }^{\circ} \mathrm{C}$ em atmosfera oxidativa, observa-se um segundo pico à direita da curva da derivada, correspondente à oxidação do carvão, fato este ausente na análise em atmosfera inerte. Em temperaturas mais elevadas e ao final do experimento $\left(900{ }^{\circ} \mathrm{C}\right)$, em atmosfera oxidante, restaram $4,8 \%$ da massa inicial correspondente às cinzas (materiais minerais), enquanto que em atmosfera inerte restaram $17,4 \%$ de massa, formados pela matéria mineral da biomassa e pelo carvão formado.

Figura 1 - Massa normalizada e sua derivada em função da temperatura em atmosfera oxidante e inerte (Rueda-Ordóñez e Tannous, 2014)

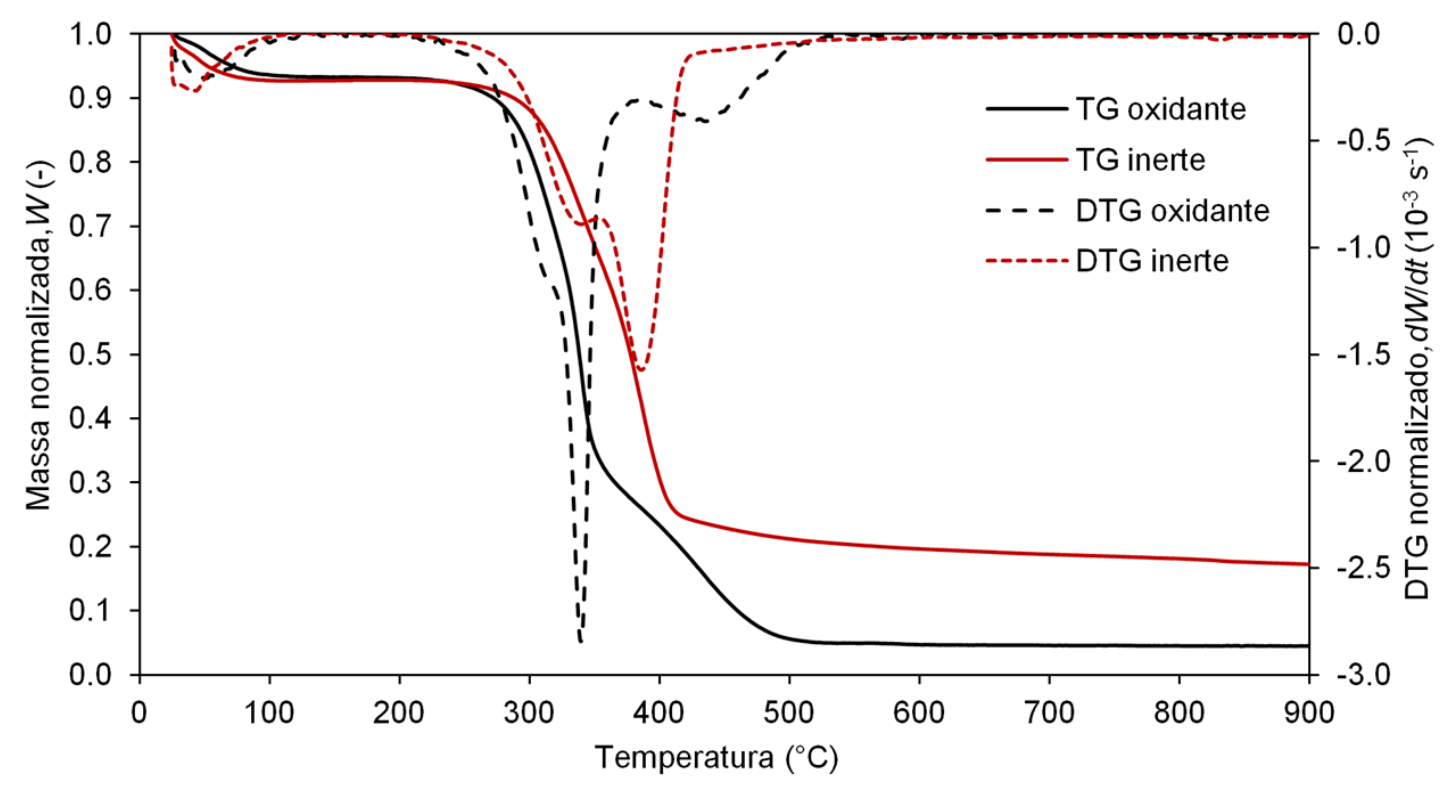

\subsection{Modelagem Cinética}

O estudo cinético em atmosfera oxidante foi realizado aplicando o modelo das reações paralelas e independentes considerando os três principais componentes da biomassa (celulose, hemicelulose e lignina). Três reações de decomposição foram consideradas, sendo uma para cada componente e três reações para a oxidação das frações de carvão formadas. As seis reações consideradas foram adotadas de primeira ordem $(n=1)$.

Na Figura 2 está apresentada a taxa de conversão em função da temperatura obtida para esse modelo em comparação aos dados experimentais. As primeiras três reações foram relacionadas à liberação de voláteis por parte da hemicelulose, HC (1), celulose, C (2) e lignina, L (3), respectivamente. Essa etapa é conhecida como pirólise oxidativa, onde as três reações subsequentes foram relacionadas à combustão das frações sólidas de carvão dos mesmos componentes, a saber: $\mathrm{HC}^{*}(4), \mathrm{C}^{*}(5)$ e $\mathrm{L}^{*}(6)$, respectivamente. Observa-se que o ajuste dos dados experimentais ao modelo pode ser considerado bastante satisfatório, o que é confirmado pelo valor do coeficiente de determinação, $\mathrm{R}^{2}=0,9848$. 
Figura 2 - Taxa de conversão teórica e experimental em função da temperatura

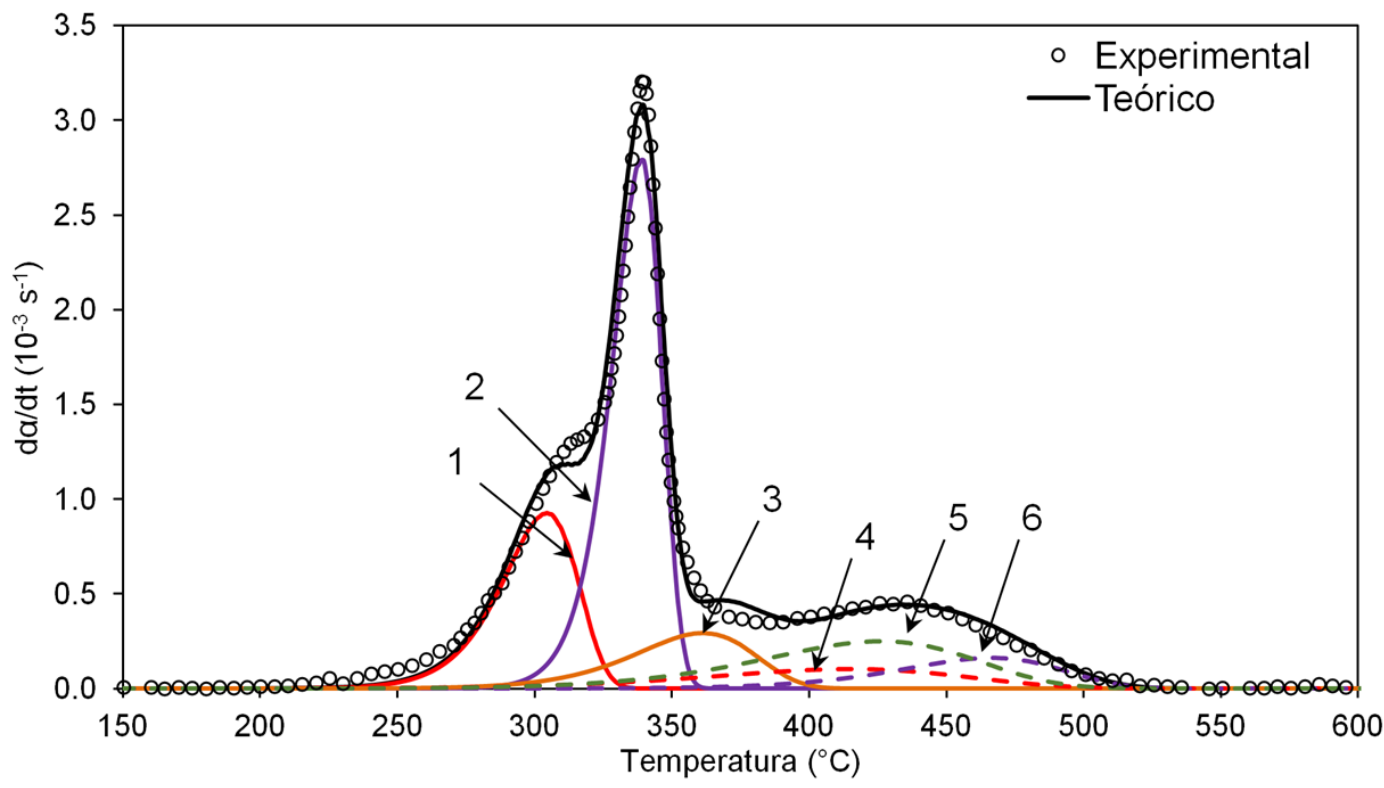

Modelo de seis reações paralelas e independentes: frações pirolisáveis [HC (1), C(2), L (3)], frações não-pirolisáveis [HC* (4), $\mathrm{C}^{*}(5)$ e $\left.\mathrm{L}^{*}(6)\right]$

$\mathrm{Na}$ Tabela 1 estão apresentados os parâmetros cinéticos obtidos para $\mathrm{HC}, \mathrm{C}$, e L das frações pirolisáveis e não pirolisáveis para atmosfera oxidante. Observa-se que houve uma diminuição de todos os parâmetros devido à diminuição das taxas de conversão referente às frações não pirolisáveis (Figura 2).

Tabela 1 - Parâmetros cinéticos obtidos para a palha de cana-de-açúcar $\left(\mathrm{d}_{\mathrm{p}}=510,31 \mu \mathrm{m}\right)$

\begin{tabular}{|c|c|c|c|c|c|c|}
\hline \multicolumn{7}{|c|}{ Atmosfera oxidante } \\
\hline & \multicolumn{3}{|c|}{ Frações pirolisáveis } & \multicolumn{3}{|c|}{ Frações não-pirolisáveis } \\
\hline & $\mathrm{HC}$ & $\mathrm{C}$ & $\mathrm{L}$ & $\mathrm{HC}^{*}$ & $\mathrm{C}^{*}$ & $\mathrm{~L}^{*}$ \\
\hline $\mathrm{Ea}(\mathrm{kJ} / \mathrm{mol})$ & 164 & 316 & 142 & 93 & 127 & 132 \\
\hline $\log A\left(s^{-1}\right)$ & 12,9 & 25,2 & 9,9 & 5,2 & 7,3 & 7,2 \\
\hline Fração & 0,19 & 0,37 & 0,09 & 0,10 & 0,09 & 0,16 \\
\hline $\mathrm{n}$ & 1 & 1 & 1 & 1 & 1 & 1 \\
\hline \multicolumn{7}{|c|}{ Atmosfera inerte (Rueda-Ordóñez e Tannous, 2014) } \\
\hline & \multicolumn{2}{|c|}{$\mathrm{HC}$} & \multicolumn{2}{|c|}{$\mathrm{C}$} & \multicolumn{2}{|c|}{$\mathrm{L}$} \\
\hline $\mathrm{Ea}(\mathrm{kJ} / \mathrm{mol})$ & \multicolumn{2}{|c|}{129} & \multicolumn{2}{|c|}{215} & \multicolumn{2}{|c|}{155} \\
\hline $\log A\left(s^{-1}\right)$ & \multicolumn{2}{|c|}{9} & \multicolumn{2}{|c|}{15} & \multicolumn{2}{|c|}{9} \\
\hline Fração & \multicolumn{2}{|c|}{0,33} & \multicolumn{2}{|c|}{0,55} & \multicolumn{2}{|c|}{0,12} \\
\hline $\mathrm{n}$ & \multicolumn{2}{|c|}{1} & \multicolumn{2}{|c|}{1} & \multicolumn{2}{|c|}{5} \\
\hline
\end{tabular}

Com relação ao comparativo entre as frações pirolisáveis e aquelas obtidas em atmosfera inerte (três reações paralelas e independentes), nota-se que as energias de ativação e o fator pré-exponencial foram mais elevados para a hemicelulose e celulose. No entanto, para o caso da lignina, os parâmetros cinéticos correspondem a uma reação mais definida numa faixa de temperatura menor do que em atmosfera inerte. 


\section{CONCLUSÕES}

A decomposição térmica da palha de cana em atmosfera oxidante apresentou três regiões principais: perda de umidade, liberação de materiais voláteis e oxidação do carvão. Em comparação à decomposição em atmosfera inerte, observou-se a ocorrência em temperaturas mais baixas e a formação de dois picos de perda de massa ao invés de apenas um, sendo o segundo pico, correspondente à oxidação do carvão.

O modelo das reações paralelas e independentes mostrou-se capaz de representar a cinética da decomposição da palha de cana-de-açúcar em atmosfera oxidante, obtendo-se um ajuste de $\mathrm{R}^{2}=0,9848$. Os parâmetros cinéticos obtidos em atmosfera oxidante foram maiores do que aqueles obtidos em atmosfera inerte, devido ao aumento da temperatura causado pelas reações de oxidação dos voláteis liberados. Devido à taxa de reação de decomposição em atmosfera oxidante ser maior, o uso de reações de primeira ordem forneceu uma melhor descrição do fenômeno. No entanto, é necessário ampliar a análise utilizando outras taxas de aquecimento, para assim determinar valores de parâmetros cinéticos que se ajustem para todas as condições.

\section{AGRADECIMENTOS}

Os autores agradecem ao CNPq e à CAPES pelo apoio financeiro concedido.

\section{REFERÊNCIAS}

ANCA-COUCE, A.; ZOBEL, N.; BERGER, A.; BEHRENDT, F. Smouldering of pine wood: kinetics and reaction heats. Combust. Flame., v. 159, p. 1708-1719, 2012.

AMUTIO, M.; LOPEZ, G.; AUGUADO, R.; ARTETXE, M.; BILBAO, J.; OLAZAR, M. Kinetic study of lignocellulosic biomass oxidative pyrolysis. Fuel, v. 95, p. 305-311, 2012.

BASU, P. Biomass gasification and pyrolysis: Practical design and theory. Elsevier: Kidlington, 2010.

BILBAO, R.; MASTRAL, J. F.; ALDEA, M. E.; CEAMANOS, A. J. Kinetic study for the thermal decomposition of cellulose and pine sawdust in an air atmosphere. J. Anal. Appl. Pyrolysis, v. 39, p. 53-64, 1997.

ÓRFÃO, J. J. M.; ANTUNES, F .J. A.; FIGUEIREDO, J. L. Pyrolysis kinetics of lignocellulosic materialsthree independent reaction model. Fuel, v. 78, p. 349-358, 1999.

RUEDA-ORDÓÑEZ, Y. J.; TANNOUS, K. Estudo Cinético da Pirólise de Biomassas mediante o Método das Reações Paralelas Independentes. In: Congresso Brasileiro de Análise Térmica e Calorimetria, 9, 2014, Serra Negra., SP.

SAI, H. H.; HUSSAIN, A.; SALEMA, A. A.; ANI, F. N. Pyrolysis and Combustion Kinetics of Date Palm Biomass Using Thermogravimetrics Analysis. Bioresour. Technol., v. 118, p. 382-389, 2012.

SENNECA, O.; CHIRONE, R.; SALATINO, P. Oxidative Pyrolysis of Solid Fuels. J. Anal. Appl. Pyrolysis, v. 71, p. 959-970, 2004. 